\title{
13 Limitationen und Ausblick
}

Für die Realisierung der Videostudie „Argumentative Lehr-Lern-Prozesse“ wurde auf eine Gelegenheitsstichprobe rekurriert. Die Teilnahme der Lehrpersonen war freiwillig, sodass Selektionseffekte nicht ausgeschlossen werden können. Zudem basiert die Untersuchung auf einem Unterrichtsinhalt, der Parteiverbotsthematik in der wehrhaften Demokratie, der zum Erhebungszeitpunkt aktuell und öffentlich diskutiert wurde. Replikationsstudien wären daher wünschenswert, um abzusichern, dass die berichteten Befunde nicht durch die Thematik bedingt sind.

Hinsichtlich der Wahrscheinlichkeit einer Änderung der persönlichen Position der Schüler/-innen sollten zukünftige Studien auch die Intensität der persönlichen Überzeugung erheben. Man kann annehmen, dass es für die Änderungswahrscheinlichkeit der eigenen Position und für die Art der Aufnahme neuer Informationen relevant ist, wie stark Schüler/-innen von ihrem Standpunkt überzeugt sind. Interessant wäre es, mittels Follow-up-Designs zu untersuchen, wie nachhaltig sich die Positionsänderung der Schüler/-innen gestaltet.

In dieser Forschungsarbeit lag der Fokus auf einem moderat kompetitiven Diskussionsformat. Zukünftige Forschung sollte argumentative Muster in stärker kooperativen Formaten in den Blick nehmen. So könnte ein weiterer Aspekt der im theoretischen Teil der Arbeit begründeten Wirkungen des Diskussionsformats auf die Art des argumentativen Diskurses geprüft werden. Des Weiteren wurden in dieser Studie keine Lernergebnisse infolge der Diskussionen untersucht. Für die Politikdidaktik sind zukünftig Studien wichtig, die die Art des argumentativen Diskurses mit Lernergebnissen in Verbindung setzen. Im Bereich der Theoriebildung ist weitgehend offen, welche konkreten Lernergebnisse in Bezug auf die Politikkompetenz durch argumentativen Diskurs im Unterricht gefördert werden können. Es ist jedoch anzunehmen, dass nicht jedes Diskussionsformat gleichermaßen zur Förderung jeder Dimension der Politikkompetenz geeignet ist. Schließlich wäre es für die Förderung des Argumentierens im Unterricht wichtig, mehr darüber zu erfahren, wie sich Argumentationskompetenz im Zeitverlauf entwickelt. Theoretische Annahmen darüber, wie das Anspruchsniveau kontroverser Diskussionen in verschiedenen Jahrgangsstufen gesteigert werden kann, wären für die Politikdidaktik wünschenswert. 